\title{
Impact of the Microstructure of Polymer Drag Reducer on Slick-Water Fracturing
}

\author{
Zhi-yu Liu, ${ }^{1}$ Fu-jian Zhou, ${ }^{1,2}$ Hong-yan Qu, ${ }^{1,2}$ Zhao Yang, \\ Yu-shi Zou, ${ }^{1,2}$ and Dao-bin Wang ${ }^{1}$ \\ ${ }^{1}$ The Unconventional Natural Gas Institute, China University of Petroleum, Beijing 102249, China \\ ${ }^{2}$ State Key Laboratory of Petroleum Resources and Prospecting, China University of Petroleum, Beijing 102249, China \\ Correspondence should be addressed to Fu-jian Zhou; zhouf@cup.edu.cn
}

Received 1 June 2017; Revised 7 August 2017; Accepted 21 August 2017; Published 27 September 2017

Academic Editor: Zhien Zhang

Copyright (C) 2017 Zhi-yu Liu et al. This is an open access article distributed under the Creative Commons Attribution License, which permits unrestricted use, distribution, and reproduction in any medium, provided the original work is properly cited.

Many studies have focused on the drag reduction performance of slick-water, but the microdrag reduction mechanism remains unclear since the microstructure of the drag reducer and its effect on this mechanism have not been well studied. In this study, the microstructure of the drag reducer in slick-water was effectively characterized by transmission electron microscopy. The viscoelasticity and drag reduction performance of the drag reducer with different microstructures were then investigated. Further, the effects of the microstructure of the drag reducer on the viscoelasticity and drag reduction performance of slick-water were analyzed. The results demonstrated that the viscoelasticity of slick-water is governed by the microstructure of the drag reducer, which exhibits a network structure. In addition, the drag reduction performance is related to the viscoelasticity. At low flow rates, the drag reduction performance is dominantly influenced by viscosity, whereas, at high flow rates, it is governed mainly by elasticity. Furthermore, the drag reducer with a uniformly distributed network structure exhibits the most stable drag reduction performance. This drag reducer was used in a field test and the obtained results were consistent with those of a laboratory experiment.

\section{Introduction}

Slick-water has become the most widely used fracturing stimulation fluid for shale owing to its outstanding drag reduction performance [1-4]. However, the drag reduction mechanism of slick-water remains unclear $[5,6]$. In the early days, drag reduction was attributed to the pseudoplasticity of fluid $[7,8]$. However, with the development of non-Newtonian fluid mechanics, this theory was believed to be inaccurate $[9,10]$. De Gennes [11] proposed that the stretching polymer molecules absorb part of the kinetic energy of the turbulent vortex and that the interaction between the polymer and the turbulence inhibits the turbulent flow, which, in turn, leads to a decrease in energy loss [11, 12]. On the basis of these theories, many researchers investigated the relationship between polymer viscoelasticity and the turbulent flow structure and validated the theory of viscoelastic drag reduction. Dimitropoulos and Angelis found that the generation and dissipative terms of turbulent energy monotonically decrease with an increase in polymer viscoelasticity [13-15]. Chemloul reported that part of the kinetic energy of tube flow was transferred to the viscoelastic polymer, rather than being entirely delivered to the turbulent fluctuation $[16,17]$.

The viscoelasticity of slick-water is the main cause of drag reduction, but the mechanism of viscoelasticity of a polymer remains unclear. Some researchers insisted that the viscoelasticity of a polymer is caused by the tensile deformation of the polymer chain under the action of external forces [18, 19]. Some others suggested that the viscoelasticity of a polymer is caused by the flexibility of the molecular chain. The more flexible the molecular chain, the more viscoelastic the polymer. Others reported that the viscoelasticity is attributed to the normal stress difference between the shear and tensile stresses of polymer molecules [20, 21].

The polymer microstructure has important effects on the viscoelasticity of the fracturing fluid, but the relationship between the drag reduction mechanism and the microstructure of the polymer drag reducer has not yet been directly 
established [22]. Some researchers revealed the relationship between the relative molecular mass of the polymer and the drag reduction performance $[23,24]$, suggesting that the drag reduction ratio has a linear relationship with the polymer molecular weight. Others took the space structure into consideration, reporting that the molecular chain length and flexibility have positive effects on the drag reduction performance [22, 25]. McCormick proposed that, in addition to the relative molecular mass, the interaction between polymers plays an important role in drag reduction [22, 26, 27]. In addition, some researchers clarified the size scale of the drag reducer microstructure and confirmed the existence of the network structure by electron microscopy methods $[28,29]$.

Previous research indicates that a certain microstructure of the drag reducer has positive effects on the drag reduction performance; however, the relationship between the drag reduction mechanism and the microstructure of the drag reducer has not been well studied. In this work, the microstructure of the drag reducer was effectively characterized by transmission electron microscopy (TEM). Further, the effects of the drag reducer microstructure on the viscoelasticity of slick-water were analyzed on the basis of the theory of viscoelastic drag reduction. Additionally, the effects of variations in the microstructure on the drag reduction performance were revealed through a field test and laboratory experiment of drag reduction.

\section{Experiments}

The drag reducer used in the experiment was acquired from Shengli Chemical Co., Ltd. (commercial name: DR-800). It is a high-molecular-weight, anionic, and water-soluble copolymer that is synthesized from a mixture of acrylamide (AM), acrylic acid (AA), 2-acrylamido-2-methylpropanesulfonic acid (AMPS), and butyl acrylate (BA) in aqueous solution by the polymerization process. The acquired drag reducer was in emulsion form.

2.1. Characterization of Drag Reducer Microstructure. The microstructures of the drag reducer with different concentrations in the range of $0.01 \%-0.15 \%$ were analyzed by TEM. The FEI Tecnai G2 F20 TEM apparatus, which has better precision and a brighter observation zone, was used to analyze the original features.

A drop of slick-water sample was placed on 230-mesh copper grids coated with a Formvar film. Excess water was removed with filter paper. After removal of excess staining liquid with filter paper, the samples were kept to dry at room temperature for TEM observations. Note that, during observation of the samples, the magnification must be increased gradually from low to high.

2.2. Viscoelasticity Test of Drag Reducer. In the context of a frequency sweep test, the energy storage coefficient of slickwater is defined as the energy stored by the drag reducer in the slick-water under alternating stress; it is represented by the elastic modulus and denoted as $G^{\prime}$. Similarly, the energy dissipation coefficient of slick-water is defined as the energy consumed by the drag reducer in the slick-water under alternating stress; it is represented by the viscous modulus and denoted as $G^{\prime \prime}$.

$G^{\prime}$ and $G^{\prime \prime}$ of slick-water with different microstructures of the drag reducer were measured at various constant frequencies by using the rheological module of the HAAKE MARS III rheometer (Thermo Scientific, Germany). All measurements in the experiments were performed under the following conditions of the key parameters: test temperature of $25^{\circ} \mathrm{C}$, frequency range of $0.01-10 \mathrm{~Hz}$, a maximum shearing rate of $3000 \mathrm{~s}^{-1}$, and a parallel-plate assembly with a gap of $1 \mathrm{~mm}$. The variations of $G^{\prime}$ and $G^{\prime \prime}$ with frequency for the different slick-water samples indicated that the microstructure of the drag reducer affects the viscoelasticity of slick-water.

2.3. Drag Reduction Ratio of Slick-Water. The drag reduction performance of slick-water can be evaluated from the pressure difference under certain flow rates or from the flow rate difference under certain pressures. However, the former approach is used more commonly than the latter one. The effectiveness of a drag reducer is usually evaluated using the percentage of drag reduction (\% DR), which is defined as

$$
\mathrm{DR} \%=\frac{\Delta P_{0}-\Delta P_{\mathrm{DR}}}{\Delta P_{0}} \times 100,
$$

where $\mathrm{DR} \%$ is the drag reduction ratio, \%; $\Delta P_{0}$ is the pressure difference with clean water; and $\Delta P_{\mathrm{DR}}$ is the pressure difference with slick-water.

The drag reduction ratio was evaluated using a pipeline friction testing system, where the pipeline had an internal diameter of $6 \mathrm{~mm}$ and length of $3 \mathrm{~m}$. Slick-water was pumped into the pipeline by means of a low-shear progressing cavity pump, where the liquid flow rate was controlled by varying the pump speed. The pressure drop was measured along a $2.5 \mathrm{~m}$ length in the middle of the pipeline by using a pressure transducer. The drag reduction ratio of slick-water was obtained by comparing the frictional pressure drops of clean water and slick-water at the same flow rate.

Experiments were conducted at laboratory temperature. The variations of the drag reduction ratios of slick-water with different microstructures of the drag reducer with a change in the flow rate were recorded in order to evaluate the effects of the drag reducer microstructure on the drag reduction performance of slick-water.

\section{Results and Discussions}

3.1. Characterization of Micronetwork Structure of Drag Reducer. The microstructures of the drag reducer with different concentrations were compared. The TEM results reveal that the microstructure configuration is mainly of two types, regular and irregular, as shown in Figure 1 . The regular microstructure typically has a uniform network structure when the drag reducer concentration is higher than $0.05 \%$ (vol\%), whereas it typically has nonuniform polygonal network units when the drag reducer concentration is reduced to $0.03 \%$. In contrast, irregular microstructures have fewer network units and contain structures of other shapes when the drag reducer concentration is as low as $0.01 \%$. 


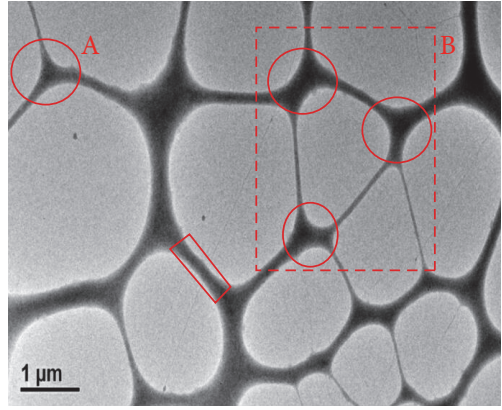

(a) Uniformly distributed network

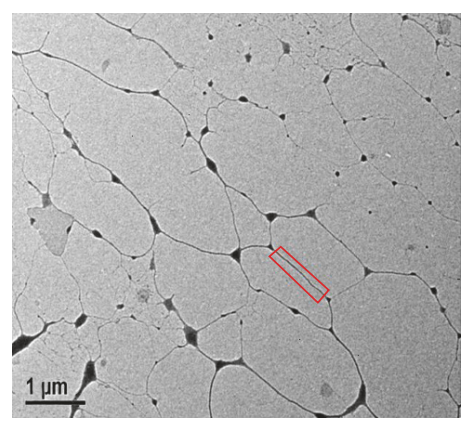

(b) Nonuniform polygonal network

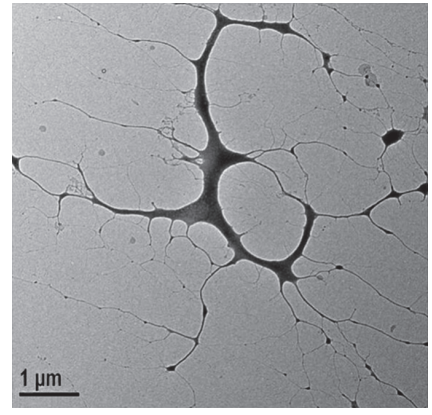

(c) Tree skeleton structure

FIgURE 1: Comparison of different microstructures of polymer drag reducer.

The network structure is formed by intertwined polymers, and the properties of the network are influenced by multiple factors. In this study, the regular microstructure is identified by the shape and size of the network unit, the number of network nodes, and the strength of the skeleton.

3.1.1. Shape and Size of Network Structure. In the regular microstructure, the network units are uniformly distributed and they have similar sizes, as shown in Figure 1(a): each unit is nearly round and the diameter of the units is $1 \mu \mathrm{m}$ on average. In contrast, in the irregular microstructure, the network units are dispersed and they have different sizes, as shown in Figure 1(b). In this case, the network units are polygonal and their diameters are different. The irregular microstructure often has a tree skeleton structure, as shown in Figure 1(c).

The drag reduction performance is strongly related to the size of the network units. The size of the network units changes considerably with a change in the internal stress during the flow of the fracturing fluid, which results in energy storage and dissipation. As a result, if the size of each unit is uniform, the slick-water becomes more elastic than viscous, which leads to high mobility and drag reduction performance. In contrast, the performance of the drag reducer is poor if the network units are either too large or too small; this is because the slick-water becomes more viscous if the network units are too small and shear failure occurs owing to a lack of network stability if the network units are too large.

For regular microstructures, the shape of the network unit is influenced mainly by the stress condition and the number of nodes. For the same number of nodes, a uniformly distributed unit has better energy storage capability than a nonuniform polygonal unit; this is because size changes of the network units are easier and mobility is better in the latter case and the drag reducer tends to be linear under a certain stress.

3.1.2. Thickness of Skeleton Wall. The network unit is composed of network nodes and skeleton walls. The strength of the network largely depends on the thickness of the skeleton wall (shown in Figures 1(a) and 1(b) and indicated by small rectangular boxes), where the skeleton walls are cemented between adjacent network units. The skeleton wall thicknesses in the network units in Figures 1(a) and 1(b) are $10 \mathrm{~nm}$ and $100 \mathrm{~nm}$, respectively; this results in different strengths of these network structures. The strength of the entire network is low if the wall is too thin but the mobility is reduced if the wall is too thick; both these scenarios result in a decreased drag reduction ratio. As shown in Figure 1(c), irregular microstructures have low stability and poor energy storage and viscosity-enhancing capabilities because of the substantial variation in the thickness of the tree skeleton wall.

3.1.3. Number of Network Nodes. A network node connects at least three network units with a shared skeleton wall. In Figure 1, node A connects three networks units, and in rectangular area $\mathrm{B}$, three nodes encompass a network unit. The number and size of nodes have a direct influence on the strength of the skeleton structure. The larger the nodes and the higher their number, the higher the number of large network units; this leads to large variations in the elasticity and viscosity of the fracturing fluid.

\subsection{Viscoelasticity of Slick-Water with Different Microstruc-} tures of Drag Reducer. The viscoelasticities of slick-water with different microstructures of the drag reducer were compared, as shown in Figure 2. As shown in Figure 2(a), for the slick-water with the tree-structured microstructure of the drag reducer, both the viscous modulus and the elastic modulus are low and they increase gradually at low frequencies. The elastic modulus, however, is lower than the viscous modulus, and thus, this slick-water exhibits a viscous character. As the frequency increases, the elastic modulus of the slick-water continues to increase at a constant rate whereas the viscous modulus remains stable. The slick-water therefore becomes predominantly elastic. As the frequency increases further, both moduli increase remarkably, and the elastic modulus is higher than the viscous modulus. Therefore, the slick-water is predominantly elastic in nature.

The $G^{\prime}$ and $G^{\prime \prime}$ values of the slick-water remain low at low frequencies because of the relatively lower strength of the tree structure. The original microstructure changes with an increase in frequency, and this leads to a change in the viscoelasticity of the slick-water because the energy storage capability increases rapidly under the imposed stress. In addition, the manner of polymer intertwining changes, 


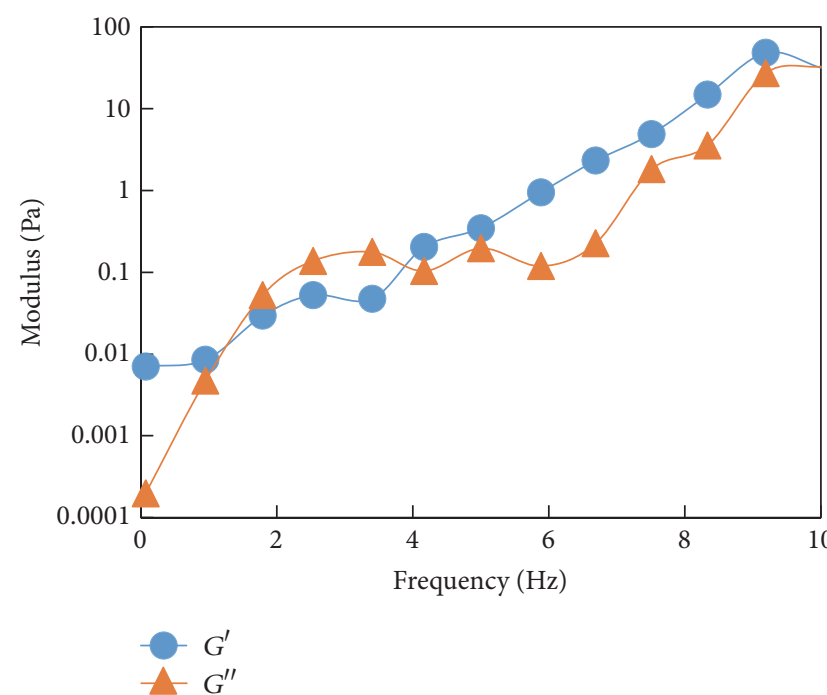

(a) Viscoelasticity of tree structure

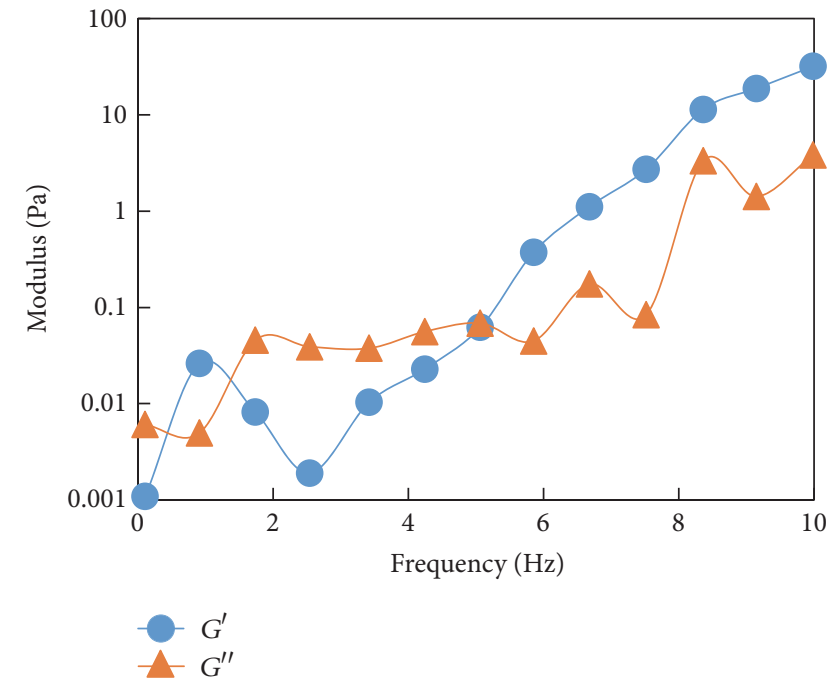

(b) Viscoelasticity of nonuniform polygonal structure

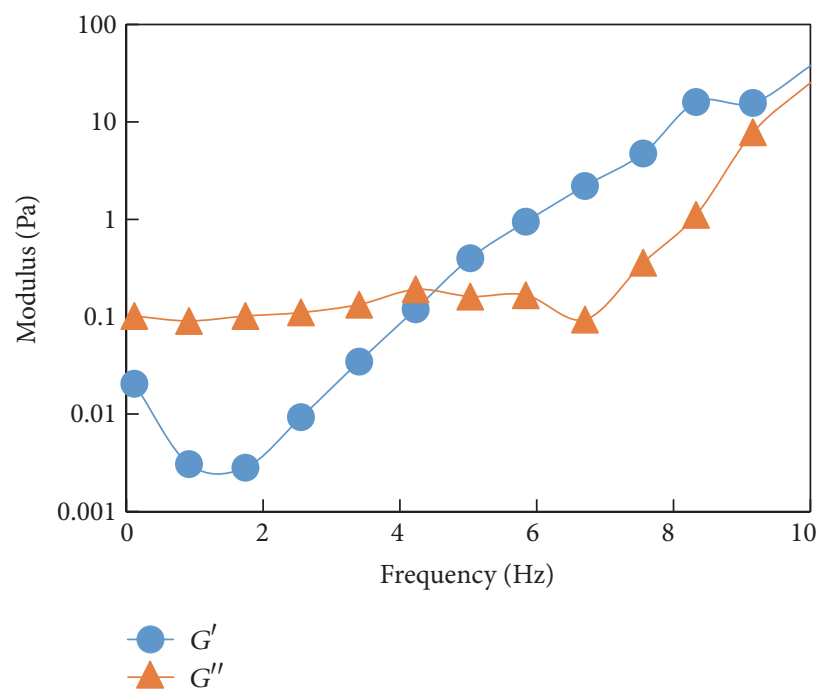

(c) Viscoelasticity of uniform structure

FIGURE 2: Comparison of viscoelasticities of different microstructures of drag reducer.

which causes an increase in the structural complexity and particle size; this eventually leads to an increase in the viscous modulus to some extent. Therefore, the drag reducer with a tree structure has poor stability.

The viscous and elastic modulus of the slick-water with a nonuniform polymer structure of the drag reducer show similar trends, as shown in Figure 2(b). However, at the frequency of $0.1 \mathrm{~Hz}$, the viscous modulus is higher than that in the case of the first microstructure. In addition, at frequencies lower than $1 \mathrm{~Hz}$, the elastic modulus decreases slightly whereas the viscous modulus increases and remains higher than the elastic modulus. The elastic modulus increases rapidly with increasing frequency and becomes higher than the viscous modulus.

The strength of the nonuniform polygonal structure of the drag reducer is relatively high because, at high frequencies, the elastic modulus is relatively higher than the viscous modulus. The elastic modulus may decrease owing to damage to some unstable, narrow network unit, which leads to storage of a smaller amount of energy in the polymer. After all the unstable structures are destroyed and new, stronger structures are formed, the elastic modulus increases rapidly. Therefore, the drag reducer with the nonuniform polygonal structure provides only a certain degree of stability because its viscoelasticity does not remain stable with changes in the microstructure; this results in a relatively unstable drag reduction performance.

Different from the cases of the previous two microstructures, the following results are obtained in the case of the uniformly distributed network structure: at frequencies lower than $1 \mathrm{~Hz}$, the elastic modulus is much lower than the viscous modulus, and with increasing frequency, the viscous modulus 


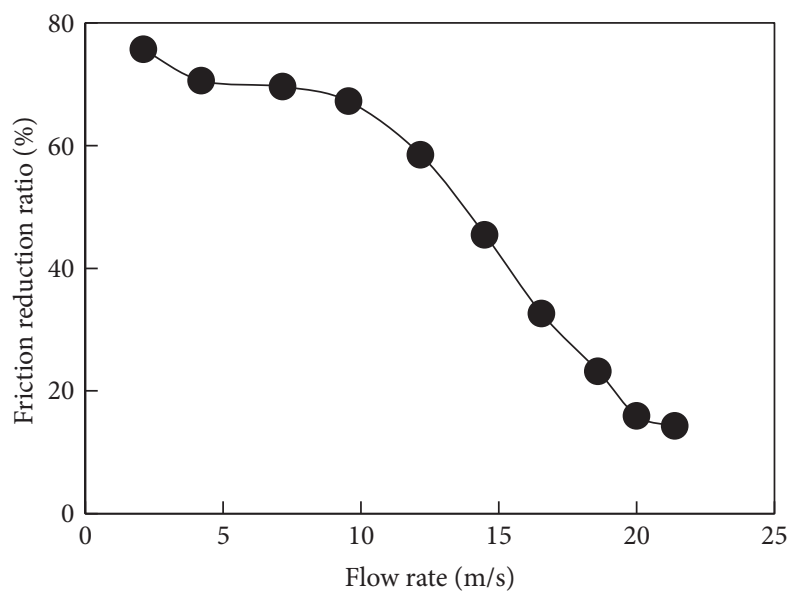

(a) Drag reduction ratio for tree structure

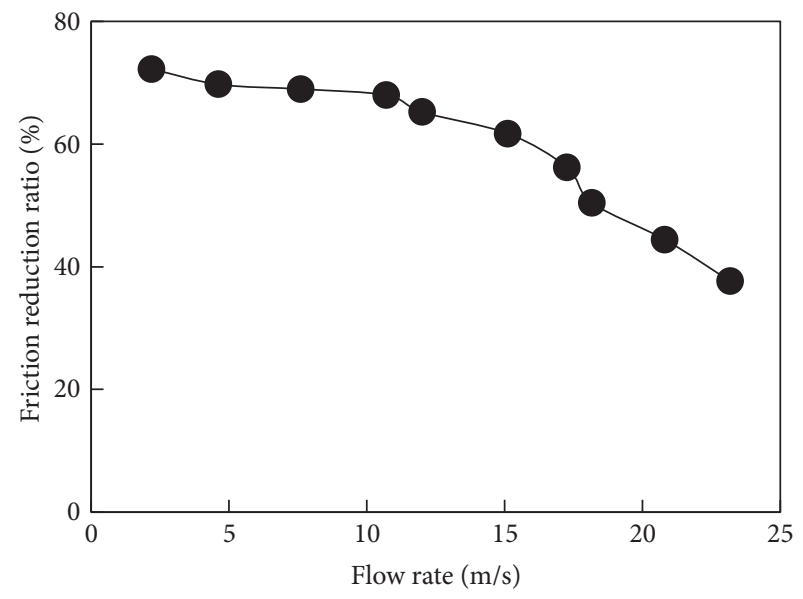

(b) Drag reduction ratio for nonuniform polygonal network structure

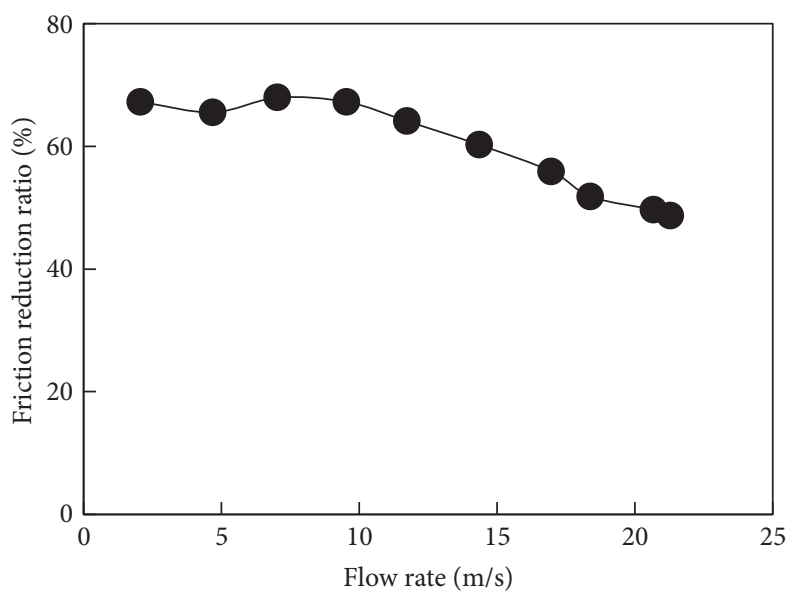

(c) Drag reduction ratio for uniform network structure

FIGURE 3: Variation of drag reduction ratio with flow rate for different microstructures.

decreases and becomes lower than the elastic modulus (see Figure 2(c)).

The distributions of the nonuniform polygonal structure and uniformly distributed structure are such that they lead to improved strength. The microstructure of the drag reducer, since the destruction of these structures at either low or high frequency, is relatively difficult. The nonuniform polygonal structure and uniformly distributed structure store larger amounts of energy, which causes an increase in the elastic modulus of the slick-water. The higher stabilities of the network units of the nonuniform polygonal and uniformly distributed structures are more beneficial for the drag reduction performance than is the poorer stability of the irregular structure, because a stable network can prevent intertwining of the polymer, which, in turn, greatly reduces the probability of formation of large particles and limits the viscosity. However, the uniformly distributed structure is more stable than the nonuniform polygonal structure, which leads to storage of a larger amount of energy and higher elastic modulus in the former.

As mentioned above, viscoelasticity is strongly related to the microstructure of the drag reducer, which, in turn, is influenced by the size and shape of the network unit and the skeleton structure. The drag reducer exhibits different microstructures in the slick-water at different flow stages, which correspondingly leads to different drag reduction mechanisms. The drag reduction performance at each stage is dependent on the balance between the viscous modulus and the elastic modulus. At low flow rates, the drag reducer forms a liquid layer against the pipeline surface, which decreases the interface drag. In contrast, at high flow rates, since the intensity of internal turbulence increases, the energy consumed within the slick-water constitutes the main drag of the pipeline. Energy can be stored through deformation of the elastic structure, which decreases the thermal dissipation and consequently reduces the pipeline drag.

3.3. Drag Reduction Performance of Slick-Water with Different Microstructures of Drag Reducer. The drag reduction performances of slick-water with different microstructures of the drag reducer were compared next, as shown in Figure 3. The drag reducer microstructures with the tree structure, nonuniform polygonal structure, and uniformly distributed structure, hereafter referred to as cases (a), (b), and (c), 
respectively, show very high initial drag reduction ability, and the initial drag reduction ratio in each case is higher than approximately $70 \%$. However, in cases (a) and (b), the drag reduction ratio decreases dramatically with an increase in the flow rate, as can be seen in Figures 3(a) and 3(b), respectively; nevertheless, the decrease rates are different at different flow rates. Specifically, for both cases (a) and (b), the drag reduction ratio decreases slowly at flow rates lower than $10 \mathrm{~m} / \mathrm{s}$; it then decreases sharply and becomes $16 \%$ and $38 \%$ for cases (a) and (b), respectively, at flow rates higher than $20 \mathrm{~m} / \mathrm{s}$. In contrast, case (c) shows better performance. In this case, the drag reduction ratio increases slightly at flow rates lower than $10 \mathrm{~m} / \mathrm{s}$, and then, it decreases gradually to approximately $50 \%$ as the flow rate increases to $22 \mathrm{~m} / \mathrm{s}$.

The stability of the drag reducer in case (a) is rather poor and the drag reduction performance is sensitive to the flow rate. The high drag reduction ratio at low flow rates is attributed to the relatively good dispersity of the polymers in the slick-water. However, because of the absence of a network in the tree skeleton (i.e., case (a)), the structure can easily undergo damage at high flow rates and is therefore vulnerable to influence by stress; this is the reason why the drag reduction ratio drops rapidly at high flow rates. The irregular network structure inhibits the drag reduction performance, and the different thicknesses of the skeleton structure and the irregular network units limit the effective amount of energy that can be stored. At high flow rates, the irregular network structures undergo some damage, which results in a relatively lower drag reduction ratio.

The existence of the polygonal structure in case (b) represents a high initial drag reduction performance; however, the structure is destroyed at high flow rates owing to its poor stability. The reticular form of the uniformly distributed network structure (case (c)) is maintained even at high flow rates, which reduces the probability of polymer intertwining and prevents any further increase in viscosity. Moreover, the regular structure effectively stores the energy of the slickwater and improves its elasticity.

As can be seen in Figure 3, the results of the drag reduction ratio are consistent with the viscoelastic nature of the drag reducer microstructure. The effect of the flow rate on the drag reduction ratio is attributed to the changes in the viscoelasticity of the slick-water, which is dependent on the microstructure of the polymer drag reducer. Therefore, the microstructure of the drag reducer governs the drag reduction performance of slick-water.

\section{Field Test}

In order to compare the drag reduction ratios obtained in the laboratory experiments with those obtained in the field and to establish whether the drag reducer with a uniform network structure exhibits good drag reduction performance, a drag reducer with a uniformly distributed network structure-which had an appropriate number of nodes and skeleton thickness-was employed in test well A in Xinjiang Province. The string size of well A was $88 \mathrm{~mm}$, and the main parameters of the slick-water used in the field test were

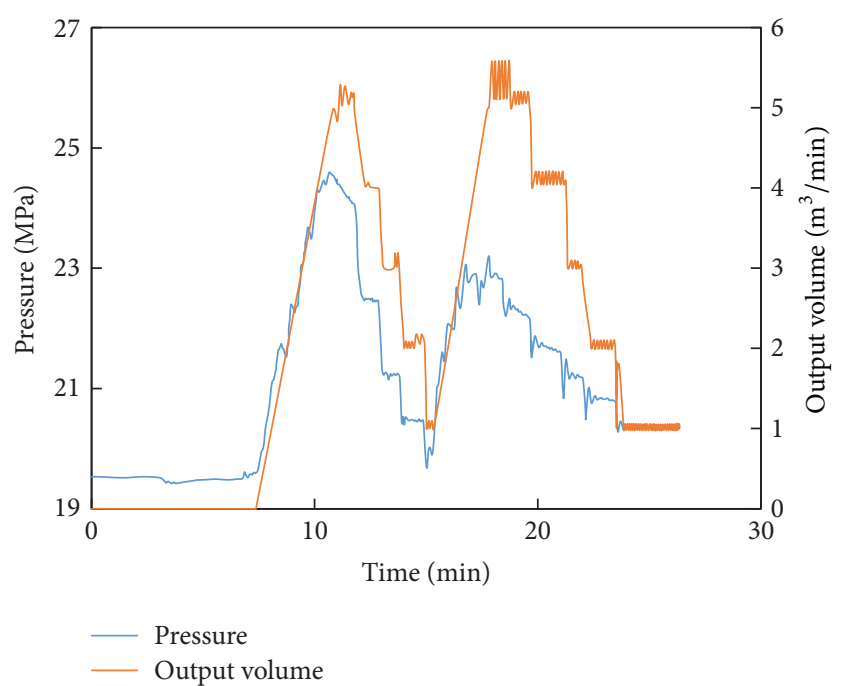

FIGURE 4: Operating pressure of drag reducer in field test in well A in Xinjiang Province.

as follows: drag reducer concentration of $0.1 \%$, viscosity of $3 \mathrm{mPa} \cdot \mathrm{s}$, and density of $1 \mathrm{~g} / \mathrm{cm}^{3}$.

For comparison with the drag reduction performance of slick-water, clean water was first injected into the pipe. The tested drag reducer was pumped into the pipe in the $33 \mathrm{rd}$ hour, and the entire process lasted for $12 \mathrm{~min}$. The total volume of the injected fluid was $34 \mathrm{~m}^{3}$. The operating pressure decreased from $24.31 \mathrm{MPa}$ to $20.48 \mathrm{MPa}$ as the output volume decreased from $5 \mathrm{~m}^{3} / \mathrm{min}$ to $1 \mathrm{~m}^{3} / \mathrm{min}$; in the same range of the output volume of the slick-water with the uniformly distributed network structure of the drag reducer, the operating pressure decreased from $22.28 \mathrm{MPa}$ to $20.38 \mathrm{MPa}$, as shown in Figure 4. Therefore, at a certain output volume, the friction in the pipeline when slick-water with the drag reducer was injected decreased in comparison to that when clean water was injected. The drag reduction ratio was calculated from the pressure change with the flow rate, and the drag reduction ratio increased with increasing output volume, as shown in Figure 5.

A comparison of the drag reduction ratio curves obtained in the field test and laboratory experiment revealed that the drag reduction ratio obtained in the field test attained a higher value than that obtained in the laboratory experiment, owing to the scale effect in the field test. Moreover, at flow rates lower than $15 \mathrm{~m} / \mathrm{s}$, no difference was found between the drag reduction ratios of the drag reducers in the field test and laboratory experiment (error of less than 10\%). However, as the flow rate increased to $20 \mathrm{~m} / \mathrm{s}$, only the drag reducer with the uniform network structure showed the same drag reduction ratio in both the field test and the laboratory experiment (the error for the tree structure was more than $20 \%)$.

The results of the field test indicate that the drag reducer with uniformly distributed network units has good drag reduction performance, which is consistent with the results of the laboratory experiment. 


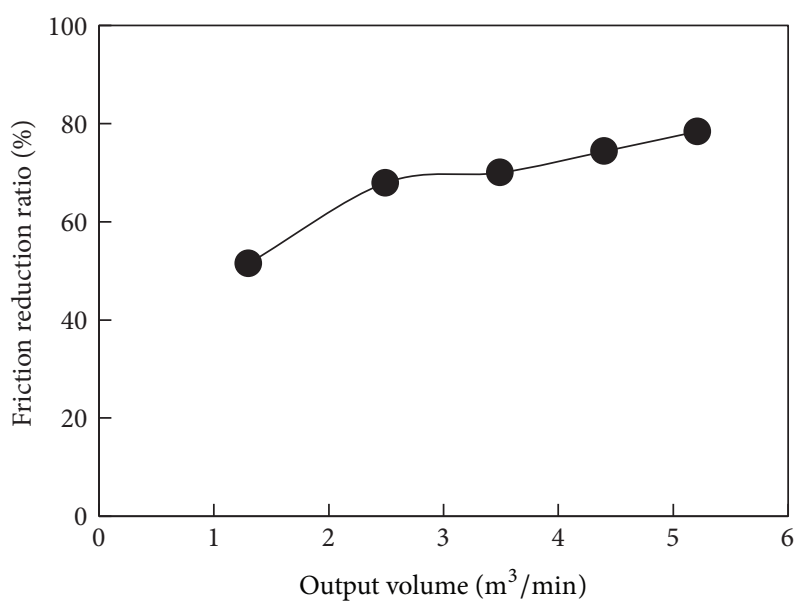

FIGURE 5: Variation in drag reduction ratio with output volume.

\section{Conclusions}

This paper proposed methods of microstructure characterization of the drag reducer in slick-water fracturing fluid. The viscoelasticity of slick-water with different microstructures of the drag reducer was analyzed to evaluate the corresponding drag reduction performance. Furthermore, the experimental results were validated by comparison with results of a field test. The following conclusions were drawn from the study.

(1) The microstructure of the drag reducer can be described using and quantitatively identified from the size and shape of the network unit, the thickness of the skeleton wall, and the number of network nodes.

(2) The viscoelasticity of slick-water is strongly related to the stability of the microstructure of the drag reducer. The viscoelasticity of slick-water at low frequencies depends mainly on the shape, size, and distribution of the network units of the drag reducer. Specifically, the elastic modulus $G^{\prime}$ is influenced mainly by the strength of the skeleton structure whereas the viscous modulus $G^{\prime \prime}$ is influenced mainly by the number of network nodes.

(3) The drag reduction performance of slick-water is dependent on the balance between the viscous modulus and the elastic modulus and is governed by the microstructure of the drag reducer. Slick-water performs better when the microstructure is regular and uniformly distributed, the number of nodes is stable, and the skeleton wall is strong.

\section{Additional Points}

Highlights. (i) The microstructure and viscoelasticity of a drag reducer are studied. (ii) Methods of microstructure characterization of the drag reducer in slick-water fracturing fluid are proposed. (iii) The viscoelasticity of slick-water is strongly related to the stability of the drag reducer microstructure. (iv) The drag reduction performance of slick-water is dependent on the balance between the viscous and elastic moduli and governed by the drag reducer microstructure.

\section{Conflicts of Interest}

The authors declare that they have no conflicts of interest.

\section{Acknowledgments}

This work is supported by the Foundation of State Key Laboratory of Petroleum Resources and Prospecting, China University of Petroleum, Beijing (no. PRP/indep-4-1314), Science Foundation of China University of Petroleum, Beijing (no. 2462014YJRC015), State Key Laboratory of Geomechanics and Geotechnical Engineering, Institute of Rock and Soil Mechanics, Chinese Academy of Sciences (no. Z014006), State Key Laboratory of Coal Resources and Safe Mining (China University of Mining and Technology) (no. SKLCRSM14KFB09), and the State Engineering Laboratory for Exploration and Development of Low Permeability Oil and Gas Fields.

\section{References}

[1] C. W. Aften, J. Paktinat, and B. O. Neil, "Critical Evaluation of Biocide-Friction Reducers Interactions Used in Shale Flowback Slickwater Fracs," in Proceedings of the SPE International Symposium on Oilfield Chemistry, The Woodlands, TX, USA, 2011 (SPE 141358).

[2] C. E. Bell and H. D. Brannon, "Redesigning Fracturing Fluids for Improving Reliability and Well Performance in Horizontal Tight Gas Shale Applications," in Proceedings of the Society of petroleum engineers hydraulic fracturing technology conference and exhibition, The Woodlands, TX, USA, 2011, (SPE 140107).

[3] Y. Cheng, "Impact of water dynamics in fractures on the performance of hydraulically fractured wells in gas-shale reservoirs," Journal of Canadian Petroleum Technology, vol. 51, no. 2, pp. 143-151, 2012.

[4] S. Rakshit, M. Jennifer L, and O. Karen E, "Laboratory Results of Proppant Transport in Complex Fracture Systems," in Proceedings of the SPE Hydraulic Fracturing Technology Conference, The Woodlands, TX, USA, 2014.

[5] M. M. Elgmati, H. Zhang, B. Bai et al., "Submicron-pore characterization of shale gas plays," in Proceedings of the Society of petroleum engineers North American unconventional gas conference and exhibition, The Woodlands, TX, USA, 2011, (SPE 144050).

[6] C. C. Hsin, H. Thomas, N. Ye X et al., "A Friction Reducer: SelfCleaning to Enhance Conductivity for Hydraulic Fracturing," in Proceedings of the SPE Annual Technical Conference and Exhibition, Amsterdam, The Netherlands, 2014, (SPE 170602).

[7] A. Toms B, "Some Observations on the Flow of Linear Polymer Solutions Through Straight Tubes at Large Reynolds Numbers," in Proceedings of the lst International Congress on Rheology, Amsterdam: Int Congress on Rheology, pp. 135-141, 1948.

[8] C. Aften and W. P. Watson, "Improved Friction Reducer for Hydraulic Fracturing," in Proceedings of the SPE Hydraulic Fracturing Technology Conference, The Woodlands, TX, USA, 2009 (SPE 118747).

[9] A. Alexander W, On the Turbulent Flow of Dilute Polymer Solutions, Calif Inst Tech, Pasadena, 1967. 
[10] S. Naveen, N. Shah S, and J. Samyak, "Friction Pressure Correlations for Newtonian and Non-Newtonian Fluids in Concentric Annuli," in Proceedings of the SPE Production Operations Symposium, pp. 16-19, Oklahoma City, Ok, USA, 2005.

[11] P. G. De Gennes, "Towards a scaling theory of drag reduction," Physica A: Statistical Mechanics and its Applications, vol. 140, no. 1-2, pp. 9-25, 1986.

[12] J. Stephane, A. Jérôme, R. Cordelier P et al., "Degradation (or lack thereof) and drag reduction of hpam solutions during transport in turbulent flow in pipelines," Oil and Gas Facilities, vol. 4, no. 1, pp. 80-92 (SPE 169699), 2015.

[13] Y. Liu T, Y. Xiang, Z. Zhao et al., "Preparation of resistance reducing agent in slick-water fracturing and characteristic research," Applied Chemical Industry, vol. 42, no. 3, pp. 484-487, 2013 (Chinese).

[14] L. C. Edomwonyi-Otu, A. H. Barral, and P. Angeli, "Influence of drag reducing agents on interfacial wave characteristics in horizontal oil-water flow," in Proceedings of the 16th International Conference on Multiphase Production Technology, Cannes, France, June 2013.

[15] L. C. Edomwonyi-Otu, M. Chinaud, and P. Angeli, "Drag Reduction in Stratified Oil-Water Flows," in Proceedings of the 9th North American Conference on Multiphase Technology, Banff, Canada, 2014.

[16] C. W. Aften, "Friction Reducers Fresh Rheological Insights Married to Performance," in Proceedings of the SPE Eastern Regional Meeting, Charleston, WV, USA, 2014, (SPE 171025).

[17] J. Paktinat, O. Neil B, and W. Aften C, "Critical evaluation of biocide-friction reducers interactions used in shale slickwater fracs," in Proceedings of the Society of Petroleum Engineers Production and Operation Symposium, Oklahoma, Ok, USA, 2011, (SPE 141356).

[18] M. C. Boyce and E. M. Arruda, "An experimental and anaiytical investigation of the large strain compressive and tensile response of glassy polymers," Polymer Engineering \& Science, vol. 30, no. 20, pp. 1288-1298, 1990.

[19] B. G. Cao, P. Y. Luo, H. B. Li et al., "Viscoelasticity and rheological behaviors of hydrophobic association polymer solution," Acta Petrolei Sinica, vol. 27, no. 1, pp. 85-88, 2006 (Chinese).

[20] G. Cao B, Y. Luo P, and G. Zhao B, "Experiment study on viscoelasticity of associative polymer solutions," Journal of Southwest Petroleum University: Science \& Technology Edition, vol. 29, no. 4, pp. 118-121, 2007 (Chinese).

[21] A. Zacharatos and E. Kontou, "Nonlinear viscoelastic modeling of soft polymers," Journal of Applied Polymer Science, vol. 132, no. 26, Article ID 42141, 2015.

[22] J. Zhu H, H. Luo J, G. Sui X et al., "Microstructure of novel polymer solution used for oil displacement," Acta Petrolei Sinica, vol. 27, no. 6, pp. 79-83, 2006.

[23] V. N. Manzhai, Y. R. Nasibullina, A. S. Kuchevskaya, and A. G. Filimoshkin, "Physico-chemical concept of drag reduction nature in dilute polymer solutions (the Toms effect)," Chemical Engineering and Processing: Process Intensification, vol. 80, pp. 38-42, 2014.

[24] Z. Fu, Y. Iwaki, M. Motozawa, T. Tsukahara, and Y. Kawaguchi, "Characteristic turbulent structure of a modified drag-reduced surfactant solution flow via dosing water from channel wall," International Journal of Heat and Fluid Flow, vol. 53, pp. 135145, 2015.

[25] S.-Q. Yang and D. Ding, "Drag-reducing flows in laminar-turbulent transition region," Journal of Fluids Engineering, Transactions of the ASME, vol. 136, no. 10, Article ID 101202, 2014.
[26] J. Zhu H, Q. Zhao C, H. Luo J et al., "Study of the microstructure of polymer molecule hydrate," Journal of Chinese Electron Microscopy Society, vol. 30, no. 6, pp. 57-63, 2013 (Chinese).

[27] J. J. Zhu, Q. Zhang G, F. Qiao G et al., "The application and research of slick-water in shale gas fracturing," Petrochemical Industry Application, vol. 32, no. 11, pp. 25-26, 2013 (Chinese).

[28] R. Zhang, B. Ye Z, and P. Luo Y, "The atomic force microscopy study on the microstructure of the polymer solution," Journal of Chinese Electron Microscopy Society, vol. 29, no. 5, pp. 75-81, 2010 (Chinese).

[29] X. Qu C, R. Li M, L. Cao X et al., "Study on microstructure of hydrophobic associating polyacrylamide by atomic force microscopy and dynamic light scattering," Polymer Bulletin, vol. 6, pp. 57-60, 2013 (Chinese). 

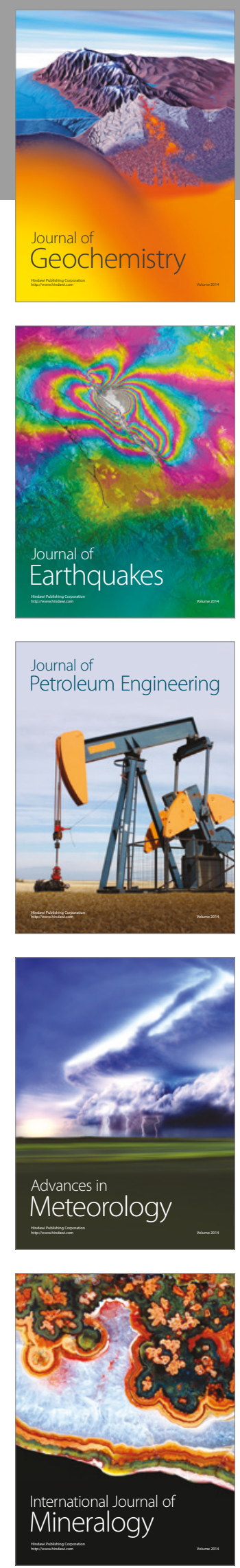
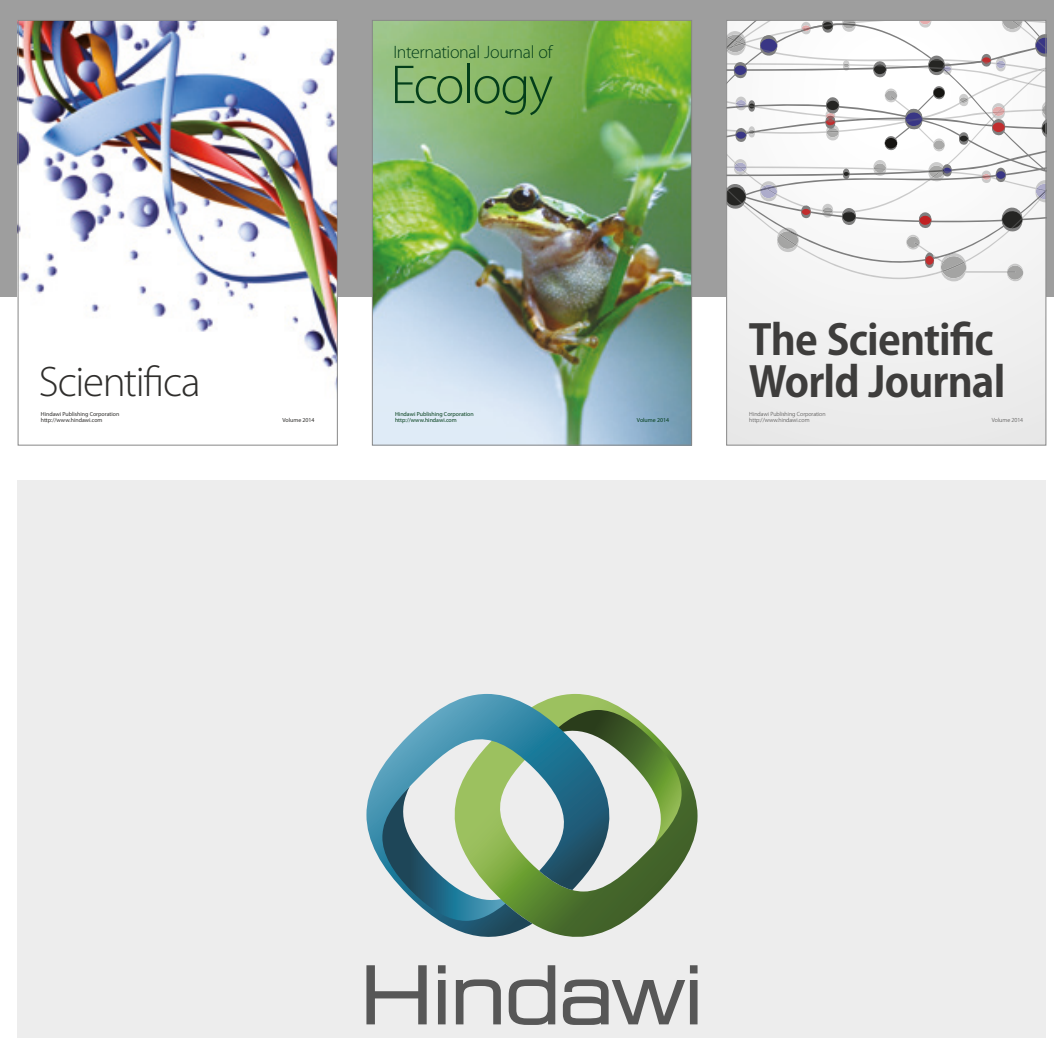

Submit your manuscripts at

https://www.hindawi.com
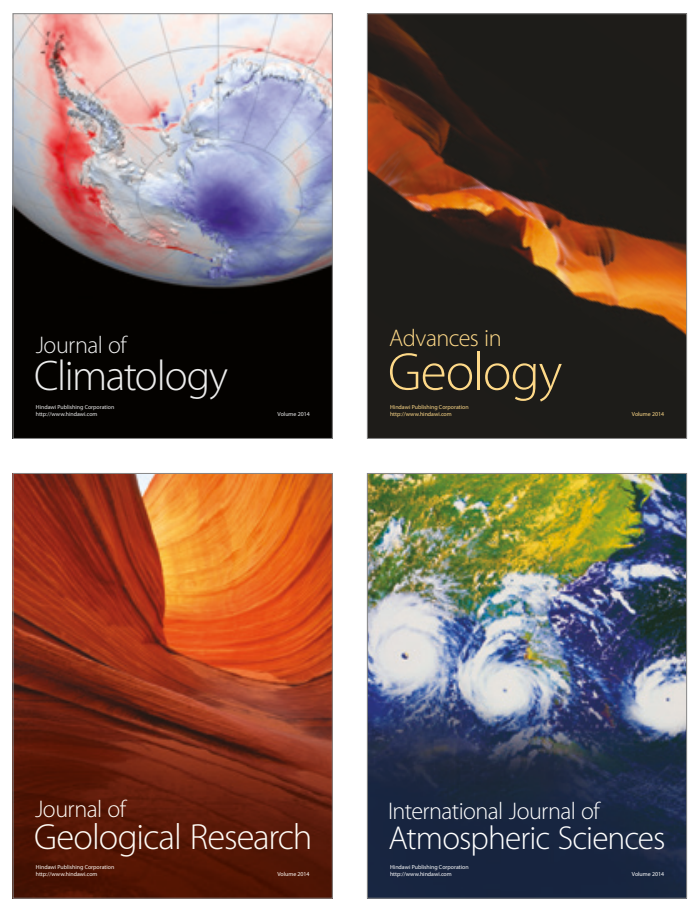

The Scientific

World Journal
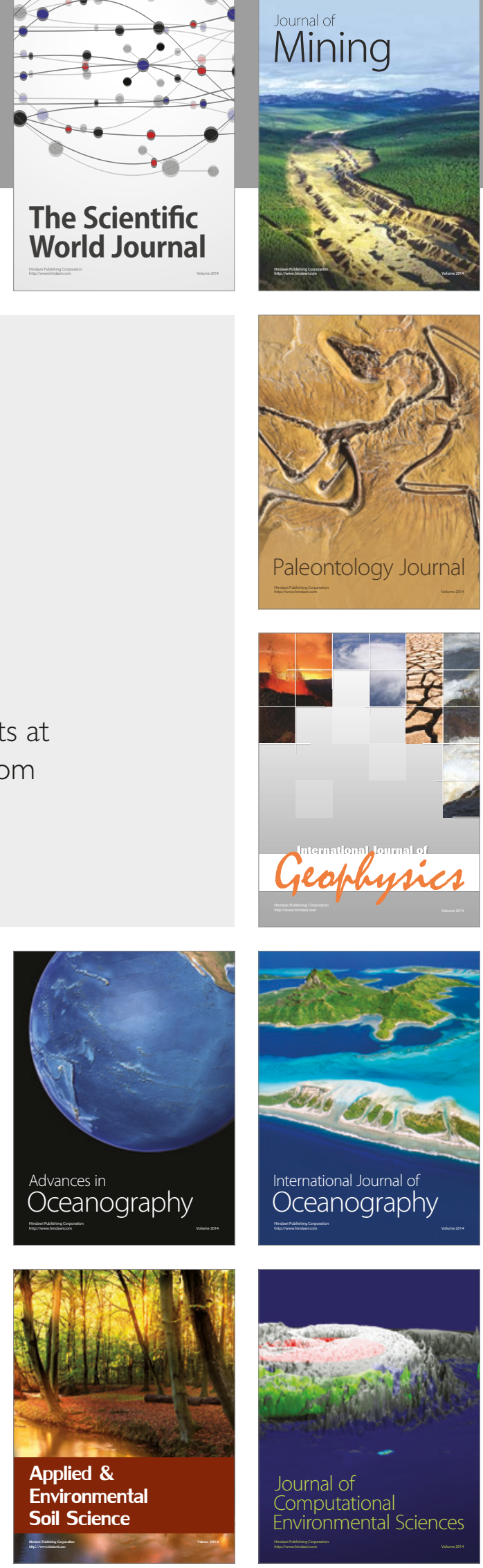